COMMUNICATIONS IN

ANALYSIS AND GEOMETRY

Volume 9, Number 2, 341-349, 2001

\title{
On Brittenham's Theorem
}

\author{
DAVID GABAI ${ }^{1}$
}

\section{Introduction.}

Theorem 0.1. Let $M, N$ be closed orientable 3-manifolds. If $f: M \rightarrow N$ is a homotopy equivalence, $\lambda, \mu$ essential laminations respectively on $N, M$ such that $f$ is transverse to $\lambda$ and pulls back to $\mu$, then $f$ is homotopic to a homeomorphism.

Mark Brittenham proved $[\mathrm{Br} 2]$ the above result under the additional hypothesis that $\lambda$ is transversely oriented. In this note we show how to remove the transverse orientability hypothesis. Removing that hypothesis is of considerable interest, since most known constructions of essential laminations on non Haken manifolds yield non transversely orientable laminations. Brittenham's theorem may play a crucial role in the resolution of the following well known Topological Rigidity Conjecture for laminar manifolds. See [Br2] or [G3] for more details.

Conjecture 0.2. If $f: M \rightarrow N$ is a homotopy equivalence, where $M$ and $N$ are closed, orientable, irreducible 3-manifolds and $N$ is laminar, then $f$ is homotopic to a homeomorphism.

Brittenham [Br2] shows that $f$ is homotopic to a homeomorphism if the preimage of each leaf is connected. In $\S 1$ we establish, using a variant of Brittenham's ideas, the connectivitiy of preimages of leaves. In $\S 2$ we give a slightly different proof that leaf preimages are connected, implies Theorem 0.1 . In $\S 3$ we suggest a new approach to proving the topological rigidity conjecture for genuinely laminar manifolds.

In light of [W] it suffices to consider the case that $N$ is non Haken. By passing to a sublamination we can assume that each leaf of $\lambda$ is dense in $\lambda$. By splitting $\lambda$ we can assume that $\lambda$ is nowhere dense in $N$.

\footnotetext{
${ }^{1}$ Partially supported by NSF Grants DMS-8902343, DMS-9200584
} 
Notation. $\quad \stackrel{\circ}{E}$ denotes the interior of $E$ and $|E|$ denotes the number of components of $E$. Basic facts and definitions about essential laminations and branched surfaces can be found in [GO].

Remark 0.3. For each leaf $L$ of $\lambda$, the mapping $f \mid f^{-1}(L): f^{-1}(L) \rightarrow L$ is proper.

Acknowledgement. The author would like the thank the referee for his/her many constructive and clarifying comments.

\section{Leaf Preimages are connected.}

Proposition 1.1. Let $M, N$ be non Haken 3-manifolds, $f: M \rightarrow N$ a homotopy equivalence, and $\lambda, \mu$ essential laminations respectively on $N, M$ such that $f$ is transverse to $\lambda$ and pulls back to $\mu$. If each leaf of $\lambda$ is dense in $\lambda$, then for each leaf $L$ of $\lambda, f^{-1}(L)$ is connected.

The next result follows by standard techniques in differential topology and foliation theory. We allow ourselves to replace the given $f$ by a new map, also called $f$ which differs from the former by a leafwise preserving homotopy.

Lemma 1.2. There exists a branched surface $B$ which carries $\lambda$ such that $f$ is transverse to $B$ and $f^{-1}(B)=C$ carries $\mu . f \mid C$ is locally an immersion except along a 1-complex $Z \subset C$ which contains the folds and branched points. The branch locus (i.e. locus of non manifold points) of $C$ is transverse to $Z$. $B$ (resp. $C$ ) has a fibred neighborhood $N(B)$ (resp. $N(C)$ ) such that $f^{-1}(N(B))=N(C)$ and if $I$ is a vertical fibre of $N(C)$, then $f \mid I$ is an embedding.

Remarks 1.3. Recall that a fold of $C$ is where $f$ is generically locally of the form $(x, y) \rightarrow(|x|, y)$. Folds may cancel at isolated points and will cross the branch locus of $B$ transversely. Branch points are locally of the form $z \rightarrow z^{2}$ near $0 \in \mathbb{C}$.

Lemma 1.4. It suffices to consider the case that $L$ is orientable.

Proof. By splitting the nonorientable leaf $L$ we obtain an orientable dense leaf $L^{\prime}$ in an essential lamination $\lambda^{\prime}$ which pulls back via $f$ to an essential 
lamination $\mu^{\prime}$ obtained by splitting $f^{-1}(L)$. If $f^{-1}\left(L^{\prime}\right)$ is connected, then so is $f^{-1}(L)$.

Orientation Conventions 1.5. We assume that $M, N$ are oriented so that $f$ is a map of degree-1. Fix an orientation on $L$. If $f$ is an immersion at $x$, then local deg $f(x)= \pm 1$ denotes the local degree of $f$ at $x$.

Lemma 1.6. Let $S=f^{-1}(L)$. $S$ has an orientation such that the following holds at each $x \in S$ for which $f$ is an immersion. Local $\operatorname{deg} f(x)=1$ at $x \in M$ if and only if $f \mid S: S \rightarrow L$ is locally degree 1 at $x \in S$.

Proof. Orient $S$ at each $x \in S$ for which $f$ is an immersion, to satisfy the conclusion of the lemma. As one crosses a fold of $f$, the local degrees of both $f$ and $f \mid S$ change, thus the partial orientation on $S$ extends to the desired orientation.

Let $y \in L$ be a regular value of $f$, which exists by Lemma 1.2. Let $\left\{x_{1}, \cdots, x_{n}\right\}=f^{-1}(y)$. Let $S_{j}$ be the component of $S$ which passes through $x_{j}$. Assume that $n$ is minimal in the sense that if $g: M \rightarrow N$ is homotopic to $f$ via a leafwise preserving homotopy, $g$ has the properties described in Lemma 1.2 and $y \in L$, then $\left|g^{-1}(y)\right| \geq n$.

Lemma 1.7. If local deg $f\left(x_{i}\right) \neq \operatorname{local} \operatorname{deg} f\left(x_{j}\right)$, then $S_{i} \neq S_{j}$.

Proof. Suppose that local $\operatorname{deg} f\left(x_{i}\right) \neq \operatorname{local} \operatorname{deg} f\left(x_{j}\right)$ but $S_{i}=S_{j}$. Let $K$ be a compact, connected $\pi_{1}$-injective subsurface of $L$ such that $V^{\prime}$ a component of $f^{-1}(K)$ contains both $x_{i}$ and $x_{j}$. Let $V$ be $V^{\prime}$ together with discs in $S-\stackrel{\circ}{V}^{\prime}$ whose boundaries lie in $V . V$ is compact by Remark 0.3. A standard 2manifold argument (e.g. see [K] or [G1]), shows that $f \mid S$ can be homotoped to a map $f^{\prime}$, via a homotopy supported in a very small neighborhood $U$ of $V$, so that $f^{\prime}(U-V) \cap y=\emptyset$ and either $f^{\prime} \mid V$ is a proper immersion onto $K$ or $y \notin f^{\prime}(V)$. In either case $\left|\left(f^{\prime} \mid S\right)^{-1}(y)\right|<n$. Using the $\pi_{1^{-}}$ injectivity of $f \mid V$, such a homotopy can be achieved by cancelling folds and the homotopy can be factored as a product of homotopies supported on discs of $S$ which map into discs of $L$. Thus by the Reeb stability theorem there exists a splitting of $B$ (and hence $C$ ) and a homotopy of $M$ obtained by cancelling folds of the split $C$ which restricts to the above homotopy on 
$V$. The resulting map $g$, is leafwise homotopic to $f$, satisfies the hypothesis of the Proposition 1.1, the conclusions of Lemma 1.2 and $\left|g^{-1}(y)\right|<n$ has been reduced. This is a contradiction.

Corollary 1.8. If $f: S_{i} \rightarrow L$ is of degree $m_{i}$, then $\left|m_{i}\right|=\left|f^{-1}(y) \cap S_{i}\right|$.

Let $I$ be the vertical fibre of $N(B)$ which contains $y . f^{-1}(I)=$ $\left(I_{1}, \cdots, I_{n}\right), f \mid I_{t}$ is a homeomorphism and $f$ is locally degree $\sigma_{t}= \pm 1$ near $I_{t}$. Indices are chosen so that for each $k, x_{k} \in I_{k}$. By Lemma 1.7 if $S_{i}=S_{j}$, then $\sigma_{i}=\sigma_{j}$.

Lemma 1.9. If $J$ is a leaf of $\mu$, then for some $i, J \cap I_{i} \neq \emptyset$.

Proof. This follows from Proposition $1.1[\mathrm{Br} 2]$ and Lemma $2.2[\mathrm{Br} 2]$ which imply that the lamination which is the closure of $J$ in $\mu$ maps surjectively to $\lambda$ under $f$.

Lemma 1.10. No connected leaf $J$ of $\mu$ passes through both $I_{j}$ and $I_{i}$ where $\sigma_{i} \neq \sigma_{j}$.

Proof. We first simultaneously prove this lemma for the connected surfaces $S_{1}, \cdots, S_{n}$ defined above. Suppose that some $S_{k}$ passed through some $I_{i}$, where $\sigma_{i} \neq \sigma_{k}$ and $z_{i} \in I_{i} \cap S_{k}$. Setting $z=f\left(z_{i}\right), \mid f^{-1}(z) \cap S_{k}$ $|>| f^{-1}(y) \cap S_{k}|=| \operatorname{deg}\left(f \mid S_{k}\right) \mid$. In fact, the equality was established in Corollary 1.8 and the inequality follows from the fact that sign(local degree $\left.f\left(z_{i}\right)\right) \neq \operatorname{sign}$ (degree $f \mid S_{k}$ ). Since $n=\left|f^{-1}(z)\right|=\left|f^{-1}(y)\right|$, the previous inequality implies that for some $j,\left|f^{-1}(z) \cap S_{j}\right|<\left|f^{-1}(y) \cap S_{j}\right|$ which implies that for some $\left\{x_{p}, x_{q}\right\} \subset f^{-1}(y) \cap S_{k}$ local deg $f\left(x_{p}\right) \neq$ local deg $f\left(x_{q}\right)$ thereby contradicting Lemma 1.7 .

A similar contradiction would be obtained if some component of $S$ nontrivially intersects some $I_{j}$ but is disjoint from $f^{-1}(y)$. Thus using Lemma 1.9 we see that Lemma 1.10 holds for each component of $S$. To obtain the general case, if some connected leaf $J$ passes through $I_{i}, I_{j}$ with $\sigma_{i} \neq \sigma_{j}$, then consider an arc $\alpha$ in $J$ which connects these points. Use the density of $S$ in $\mu$ and the Reeb stability theorem applied to $\alpha$ to find a component $T$ of $S$ which hits both $I_{j}$ and $I_{i}$.

Let $P$ (resp. $Q$ ) be the sublamination of $\mu$ consisting of leaves which pass through $I_{j}$ 's where $\sigma_{j}=-1\left(\right.$ resp. $\left.\sigma_{j}=1\right)$. 
Lemma 1.11. One of $P$ or $Q$ is empty and therefore $n=$ degree $f=1$.

Proof. This follows from the fact that two essential laminations in a non Haken manifold must nontrivially intersect [Br1]. (Idea-Since leaves of an essential lamination are $\pi_{1}$-injective in the ambiant manifold, so are complementary regions. Thus if $D$ is a branched surface which carries $P$, such that all the interstitial regions of $N(D)$ are noncompact, then each complementary region $E$ of $N(D)$ is a handlebody since $M$ is non Haken. Since each of $P$ and $Q$ are compact, $Q$ can penetrate only a compact portion of the interstitial fibres of $N(D)$. Thus $Q$ embeds in a handlebody. Using the fact that efficient closed curves are $\pi_{1}$-injective, it follows that $Q$ can be isotoped off of any compressing disc and hence $Q$ embeds in a 3-ball, which implies that each leaf of $Q$ is a sphere and hence $Q$ is inessential.)

Proof of Proposition 1.1. By Lemma 1.11, $\mathrm{n}=\operatorname{deg} f=1$. If $J$ is a component of $f^{-1}(L)-S_{1}$, then $y \notin f(J)$ so $\operatorname{deg} f \mid J=0$ and hence $J \cap I_{1}=$ $\emptyset$ thereby contradicting Lemma 1.9. Thus $f^{-1}(L)$ is connected. Although our $f$ was altered in the course of the proof, the alterations were via leaf preserving homotopies. Thus the preimage of each leaf of $\mu$ is independent of the choice of $f$.

Corollary 1.12. There exists an $f$ satisfying the hypothesis and conclusions of Proposition 1.1 and Lemma 1.2 and there exists an embedded disc $E \subset B$, such that $f \mid f^{-1}(N(E)) \rightarrow N(E)$ is a homeomorphism. Here $N(E)$ denotes the I-fibres of $N(B)$ passing through $E$.

\section{Proof of Theorem 0.1.}

If $N(B)$ is the fibred neighborhood of the branched surface $B$, then $\pi$ : $N(B) \rightarrow B$ denotes the projection which contracts $I$ fibres to points. Let $B, E, \lambda, f$ be as in Corollary 1.12 and let $\mathcal{E}$ denote $\pi^{-1}(E) \cap \lambda$.

Lemma 2.1. There exists a $\lambda$-splitting $B_{1}$ of $B$ such that $K$ collapses to a 1-complex, where $K=p^{-1}(B-\stackrel{\circ}{N}(E)) \subset B_{1}$ and $p: B_{1} \rightarrow B$ is the quotient map which inverts the $\lambda$-splitting. 
Proof. There exists a $N<\infty$ such that for any $x \in L$ a leaf of $\lambda$, then $d(x, \mathcal{E})<N$. Here view $L$ as a simplicial complex, with a simplicial structure induced from one on $B$. The distance is the minimal number of 1-cells a path in $L$ must traverse to get from $x$ to $L \cap \mathcal{E}$. If no such $N$ exists, then there exists $\left\{x_{i}\right\}$ such that $d\left(x_{i}, \mathcal{E}\right) \rightarrow \infty$. If $x$ is a limit point of $\left\{x_{i}\right\}$, then the finiteness of $d(x, \mathcal{E})$ together with the Reeb stability theorem yield a contradiction.

Thus after allowing for $\lambda$-splitting, a series of elementary collapses of $B-\stackrel{\circ}{E}$ reduces $B$ to a 1 -complex. In fact by collapsing $\lambda-\mathcal{E}$ in $N(B)$ over one $\pi^{-1}(2$-simplex of $B$ ) at a time (however one may have to collapse over the same 2-simplex several times), one eventually collapses $\lambda-\mathcal{E}$ to a set which lies in the $\pi$ preimage of a 1-complex in $B$. The splitting of $B$ is done to accomplish this at the branched surface level. Finally let $B_{1}$ be this split branched surface.

Remark 2.2. $K$ could be chosen to have a nonmanifold locus consisting of arcs, which project to points under the collapse to a 1-complex.

Lemma 2.3. $f$ can be homotoped so that $f \mid N\left(f^{-1}\left(B_{1}-\stackrel{\circ}{K}\right)\right)$ is a homeomorphism onto $N\left(B_{1}-\stackrel{\circ}{K}\right)$.

Proof. Combine Corollary 1.12 and Lemma 2.1.

Lemma 2.4. After splitting $B_{1}$ to $B_{3}, f$ can be homotoped so that $f \mid$ $N\left(f^{-1}\left(B_{3}\right)\right) \rightarrow N\left(B_{3}\right)$ is a homeomorphism.

Proof. Let $A$ be a union of properly embedded $\operatorname{arcs}$ in $K$ which cut $K$ into a union of collapsable 2-complexes. If $\alpha$ is an arc in $\pi^{-1}(A) \cap \lambda$, then let $L$ be the leaf of $\lambda$ which contains $\alpha$ and let $S=f^{-1}(L)$. A homotopy of $f \mid S$, as in the proof of Lemma 1.7, can be found so that the resulting map also called $f$ satisfies $f \mid f^{-1}(\alpha)$ is a homeomorphism. Since a homotopy which straightens out $f$ near $\alpha$ can be thickened to straighten out nearby arcs, and $A$ is compact, it follows that after a splitting of $B_{2}, f$ can be deformed to be a homeomorphism except on the finite set of collapsable subcomplexes $K_{2}$ of $B_{2}$ associated to $K-A$. Each component $D$ of $\pi^{-1}\left(K_{2}\right) \cap \lambda$ is a disc as is $f^{-1}(D)=F$. Thus $f \mid F$ can be homotoped rel boundary to be a homeomorphism. Hence by compactness, after a further splitting of $B_{2}$ to 
$B_{3} f \mid f^{-1}\left(B_{3}\right) \rightarrow B_{3}$ is a homeomorphism. A further homotopy extends the homeomorphism to the fibred neighborhoods.

Proof of Theorem 0.1 .

$$
f:\left(M, N\left(f^{-1}\left(B_{3}\right)\right), M-\stackrel{\circ}{N}\left(f^{-1}\left(B_{3}\right)\right)\right) \rightarrow\left(N, N\left(B_{3}\right), N-\stackrel{\circ}{N}\left(B_{3}\right)\right)
$$

such that $f \mid N\left(f^{-1}\left(B_{3}\right)\right)$ is a homeomorphism. But

$$
f: M-\stackrel{\circ}{N}\left(f^{-1}\left(B_{3}\right) \rightarrow N-\stackrel{\circ}{N}\left(B_{3}\right)\right.
$$

is a map of handlebodies which restricts to a homeomorphism on the boundary. Thus by [W], $f \mid M-\stackrel{\circ}{N}\left(f^{-1}\left(B_{3}\right)\right.$ is homotopic to a homeomorphism rel boundary and hence $f$ is homotopic to a homeomorphism.

\section{A program for the topological rigidity of genuinely laminar manifolds.}

Recall that $N$ is genuinely laminar if it contains a genuine lamination [GK1]. A genuine lamination is an essential lamination that does not readily extend to a foliation, i.e. some closed complementary region is not an $I$-bundle. It was shown in [GK2] that if $N$ is atoroidal and genuinely laminar, then it has group negative curvature. By $[\mathrm{BM}], \tilde{M}$ naturally compactifies to a closed 3-ball $\mathbb{B}^{3}$.

Here is a program for proving the topological rigidity conjecture for genuinely laminar manifolds.

Step 1: Generalize [G4] to negatively curved 3-manifolds.

In [G4] we established the topological rigidity of hyperbolic 3-manifolds satisfying the insulator condition. There we indicated what needs to be done to generalize to manifolds with group negative curvature.

Step 2: Prove

Conjecture 3.2. Let $N$ be a closed atoroidal oriented 3-manifold. If $\lambda$ is a genuine lamination on $N$, then the lift of each leaf of $\lambda$ to $\tilde{N}$, the universal covering of $N$, extends to a continuous mapping of the closed disc into $\mathbb{B}^{3}$.

Step 3: Prove 
Conjecture 3.3. If $N$ is a closed atoroidal genuinely laminar 3- manifold, then there exists a simple closed curve $\gamma$ in $N$ which satisfies the insulator condition.

For example if $\lambda$ is the stable lamination of a pseudo-Anosov flow, then conjecturally $\gamma$ can be taken to be a closed singular orbit of the flow. If Conjecture 3.2 holds, then the various insulators will correspond to perturbations of the ideal boundaries (or some subset if they do not embed) of leaves of $\lambda$.

If Conjecture 3.2 holds, then a candidate family is obtained by first taking a simple closed curve which lies in the image in $\mathbb{S}_{\infty}^{2}$ of the ideal boundary of any leaf and then considering all of the $\pi_{1}(N)$ translates. For example, if $\lambda$ is the stable lamination of a pseudo-Anosov flow, and $J$ is a leaf on the boundary of a non I-bundle complementary region, then surely a small purturbation of the $\pi_{1}(N)$ orbit of $\partial \tilde{J}$ (or some subset if it does not embed in $\mathbb{S}_{\infty}^{2}$ ) satisfies the no trilinking condition. The open solid torus in $N$ arising from the insulator construction should contain a core isotropic to a closed (singular) orbit of the flow.

\section{References.}

[BM] M. Bestvina and J. Mess, The boundary of negatively curved groups, J. Amer. Math. Soc. 4 (1991), 469-481.

[Br1] M. Brittenham, Essential Laminations in non Haken 3-manifolds, Top. and App. 53 (1993), 317-324.

[Br2] Essential Laminations and Deformations of Homotopy Equivalences: From Essential Pullback to Homeomorphism, Top. and App. 60 (1994), 249-265.

[FO] W. Floyd and U. Oertel, Incompressible Surfaces via Branched Surfaces, Topology, 23, 117-125.

[G1] D. Gabai, The Simple Loop Conjecture, J. Diff. Geom. 21 (1985), 143-149.

[G2] Foliations and 3-Manifolds, Proc. ICM Kyoto-1990 1 (1991), $609-619$.

[G3] Problems in foliations and laminations, AMS/IP Stud. Adv. Math. 2.2 (1997), 1-33.

[G4] On the Geometric and Topological Rigidity of Hyperbolic 3Manifolds, J. Amer. Math. Soc. 10 (1997), 37-74.

[GK1] D. Gabai and W.H. Kazez, Homotopy, Isotopy and Genuine Laminations of 3-Manifolds, AMS/IP Stud. Adv. Math. 2.1 (1997), 123-138. 
[GK2] D. Gabai and W.H. Kazez, Group Negative Curvature for 3-Manifolds with Genuine Laminations, Geom. Topol. 2 (1998), 65-77.

[GO] D. Gabai and U. Oertel, Essential Laminations in 3-Manifolds, Ann. of Math. 130 (1989), 41-73.

[K] H. Kneser, Glattung von Flachenabbildungen, Math. Ann. 100 (1928), 609617.

[W] F. Waldhausen, On Irreducible 3-Manifolds which are Sufficiently Large, Annals of Math. 87 (1968), 56-88.

CALIFornia Institute of TEChNOLOGY

PASADENA, CA 91125

USA

E-mail address: Gabai@its.caltech.edu

Received February 26, 1999. 